\title{
Research on Optimization of MTI Filters Based on Zero Distribution Method
}

\author{
Dan Jin \& Hao Cha \& Runzhe Zhang \\ Naval University of Engineering, WuHan, China
}

\begin{abstract}
For the need of the clutter suppression in the simulation of radar system and the need of the improvement of the radar signal SNR to detect moving target, it analyzed the commonly used delay line cancellation in MTI. Aiming at the disadvantage and shortage, it established a geometric model based on the zero distribution method, and through comparing the Matlab simulation, it validated the feasibility and superiority of the zero distribution method.
\end{abstract}

KEYWORD: MTI; delay line cancellation; zero distribution method; model simulation

\section{GENERAL INSTRUCTIONS}

MTI refers to the technology that takes advantage of the clutter suppression filter to suppress the clutter and improves the radar signal SNR in order to detect moving target. MTI filter is used in the pulse radar with the difference of Doppler frequency between the clutter and moving targets, which makes the filter frequency response form a "notch" in the location of the clutter spectrum to suppress the clutter and let the echo of moving targets through whit the loss as small as possible or even no loss[1].

The delay line cancellation is the earliest and is one of the most commonly used MTI filters. According to the different cancellation numbers, it divided into single delay line cancellation, double delay line cancellation and multiple delay line cancellation [2]. But the delay line cancellation can only improve the zero Doppler frequency response, and to the reality of cloud clutter, the improvement is not ideal. The MTI filter based on zero distribution method can be very good to make up for the shortage.

Zero distribution method is a technology that sets a "notch" at the zero frequency response when a band-stop filter is designed. Under the environment of the application of adaptive clutter suppression, the ideal filter needed is that enables the clutter to get maximum limit in the frequency points of the clutter and has the largest flat amplitude to other frequency ranges.

\section{THE ESTABLISHMENT OF THE MATHEMATICAL MODEL}

\subsection{The single delay line cancellation}

The single delay line cancellation is shown in Figure1. It is composed of delay units (digital delay line) whose delay time is equal to the transmitted pulse repetition period PRI $\left(T_{r}\right)$ and the adder. Single delay line cancellation is often referred to as the "two impulse cancellation" or "once cancellation".

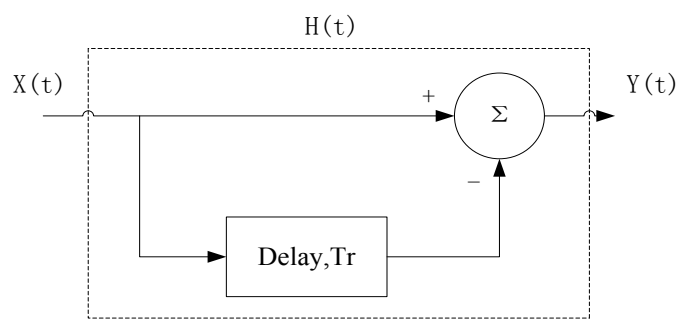

Figure 1. Single delay line cancellation

The impulse response of the cancellation is expressed as $h(t)$ and output $y(t)$ is equal to the convolution between the impulse response $h(t)$ and inpu $x(t)$. The output $y(t)$ is expressed as following

$$
y(t)=x(t)-x\left(t-T_{r}\right)
$$

The impulse response of cancellation is expressed as

$$
h(t)=\delta(t)-\delta\left(t-T_{r}\right)
$$


In the formula, $\delta(\bullet)$ is function $\delta$. Then we can obtain the Fourier transform (FT) of the $h(t)$, namely frequency response is

$$
H(w)=1-e^{-j w T_{r}}
$$

And $w=2 \pi f$.

In the $\mathrm{z}$ domain, the transfer function of single delay line cancellation is

$$
H(z)=1-z^{-1}
$$

The power gain of single delay line cancellation is

$$
\begin{aligned}
& |H(w)|^{2}=H(w) H *(w) \\
& =\left(1-e^{-j w T_{r}}\right)\left(1-e^{j w T_{r}}\right) \\
& =2\left(1-\cos w T_{r}\right)=4\left(\sin \frac{w T_{r}}{2}\right)^{2}
\end{aligned}
$$

\subsection{Double delay line cancellation}

Double delay line cancellation is shown in figure 2, and it is composed of two single delay line cancellations in cascade. Double delay line cancellation is often referred to as the "three impulse cancellation" or "secondary cancellation".

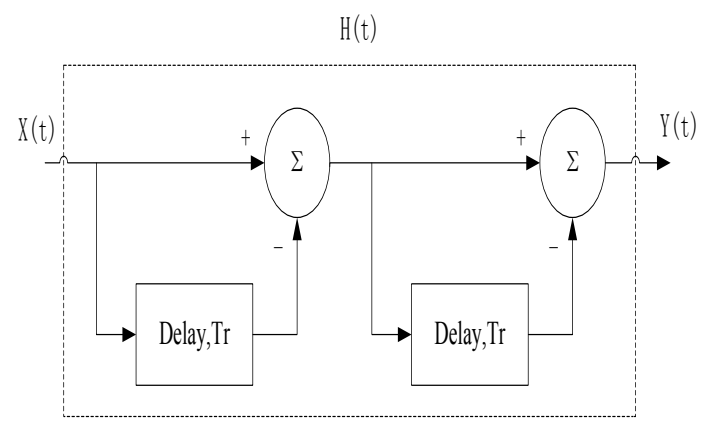

Figure 2. Double delay line cancellation

The impulse response of double delay line cancellation is expressed as

$$
h(t)=\delta(t)-2 \delta\left(t-T_{r}\right)+\delta\left(t-2 T_{r}\right)
$$
is

The power gain of double delay line cancellation

$$
|H(w)|^{2}=\left|H_{1}(w)\right|^{2}\left|H_{1}(w)\right|^{2}
$$

In the formula, $\left|H_{1}(w)\right|^{2}$ is the power gain of single delay line cancellation, and we can get

$$
|H(w)|^{2}=16\left(\sin \frac{w T_{r}}{2}\right)^{4}
$$

In the $\mathrm{z}$ domain, the transfer function of double delay line cancellation is

$$
H(z)=\left(1-z^{-1}\right)^{2}=1-2 z^{-1}+z^{-2}
$$

Single delay line cancellation is a very simple filter. Its implementation doesn't need the multiplication, each output sample only need a subtraction operation. However, compared with the ideal high-pass filter, it is only a poor approximation. Double delay line cancellation can obviously improve the notch width of the zero point Doppler, but it cannot improve the frequency response of the non-zero point Doppler frequencies. Based on the above shortcomings of delay line cancellation, we put forward the optimization design of MTI filter based on zero distribution method.

\subsection{Zero distribution method}

Zero distribution method is a technology that sets a "notch" at the zero frequency response when a bandstop filter is designed. Under the environment of the application of adaptive clutter suppression, the ideal filter needed is that enables the clutter to get maximum limit in the frequency points of the clutter and has the largest flat amplitude to other frequency ranges[3-5].

For the FIR filter of $\mathrm{N}$ order. the weight coefficient of filter is $w_{i}(i=0,1,2, \cdots$, and the frequency response function is

$$
H(f)=\sum_{i=0}^{N} w_{i} e^{-j 2 \pi f T_{i}}
$$

And $T_{0}=0, T_{i}=\sum_{k=1}^{i} T_{r, k}(i=1,2, \cdots, \mathrm{N}), T_{r, k}$ is the time interval between each pulse.

Put $H(f)$ into Taylor series at $f=f_{0}$

$$
\begin{gathered}
H(f)=H\left(f_{0}\right)+H^{\prime}\left(f_{0}\right) \cdot\left(f-f_{0}\right) \\
+\frac{H^{\prime \prime}\left(f_{0}\right)}{2 !}\left(f-f_{0}\right)^{2}+\cdots
\end{gathered}
$$

In the formula,

$$
H^{(k)}\left(f_{0}\right)=(-j 2 \pi)^{k} \sum_{i=0}^{N} T_{i}^{k} w_{i} e^{-j 2 \pi f T_{i}}
$$

When we design band-stop filter at $f=f_{0}$, the coefficient of $\left(f=f_{0}\right)^{k}$ in the Taylor series shall be 0 , namely $H^{(k)}\left(f_{0}\right)=0$. This produces $\mathrm{N}$ homogeneous linear equations of $w_{i}(i=0,1,2, \cdots, N)$.

$$
\sum_{i=0}^{N} T_{i}^{k} w_{i} e^{-j 2 \pi f_{0} T_{i}}=0, i=0,1, \cdots, N-1
$$

And $T_{i}^{0}=1, w_{0}$ is a constant, usually set to 1 . Put the formula into matrix form 


$$
A W=-w_{0} U
$$

And $W=\left[w_{1}, w_{2}, \cdots, w_{N}\right]^{T}, \quad U=[1,0, \ldots, 0]^{T}$

$$
A=\left[\begin{array}{ccc}
\mathrm{e}^{-\mathrm{j} 2 \pi \mathrm{f}_{0} \mathrm{~T}_{1}} & \cdots & \mathrm{e}^{-\mathrm{j} 2 \pi \mathrm{f}_{0} \mathrm{~T}_{\mathrm{N}}} \\
\vdots & \ddots & \vdots \\
\mathrm{T}_{1}^{\mathrm{N}-1} \mathrm{e}^{-\mathrm{j} 2 \pi \mathrm{f}_{0} \mathrm{~T}_{1}} & \cdots & \mathrm{T}_{\mathrm{N}}^{\mathrm{N}-1} \mathrm{e}^{-\mathrm{j} 2 \pi \mathrm{f}_{0} \mathrm{~T}_{\mathrm{N}}}
\end{array}\right]
$$

We can use Gauss - Jordan method to solve this equation, and get the weight coefficient of the filter.

When the stop-band is in the zero frequency, the designed filter is the biggest flat stop-band filter in the zero frequency, and $\mathrm{A}$ is the Vander-monde matrix.

$$
\mathrm{A}=\left[\begin{array}{ccc}
1 & \cdots & 1 \\
\vdots & \ddots & \vdots \\
\mathrm{T}_{1}^{\mathrm{N}-1} & \cdots & \mathrm{T}_{\mathrm{N}}^{\mathrm{N}-1}
\end{array}\right]
$$

When the zero point is single, the stop-band notch of the filter is narrow; therefore we can set a few more zero points within the stop-band to broaden its stop-band. And the length of the filter should be greater than the number of zero points.

\section{THE SIMULATION AND ANALYSIS [6]}

The normalized frequency responses of single delay line cancellation and double delay line cancellation. From the figure 3 and figure 4 , we can see that double delay line cancellation has better response than single delay line cancellation (deeper notch). Frequency response of the single delay line cancellation is rather poor, for its stop-band notch is narrow, and double delay line cancellation has better frequency response either within stop-band or passband. Double delay line cancellation can obviously improve the notch width of zero point Doppler, but it cannot improve the frequency response of nonzero Doppler.

Average repetition frequency is $100 \mathrm{~Hz}$, stagger ratio is 27:28:29, the center of the ground clutter frequency is $0 \mathrm{~Hz}$, and the spectral width is $0.64 \mathrm{~Hz}$. Use zero attribution method to design a single zero point cancellation filter of 4 impulses and the normalized frequency response is shown in figure 5 . In figure 6, we assume that the center frequency of the ground clutter is $0 \mathrm{~Hz}$, spectral width is $0.64 \mathrm{~Hz}$, the center frequency of the cloud clutter is $30 \mathrm{~Hz}$ and the spectral width is $1.4 \mathrm{~Hz}$. Use zero attribution method to design a multiple zero points cancellation filter of 6 impulses and we can get the normalized frequency response. From the figure 5 and 6 , we can get that the multiple zero points cancellation filter has a deeper and wider notch than the single one which is we expect.

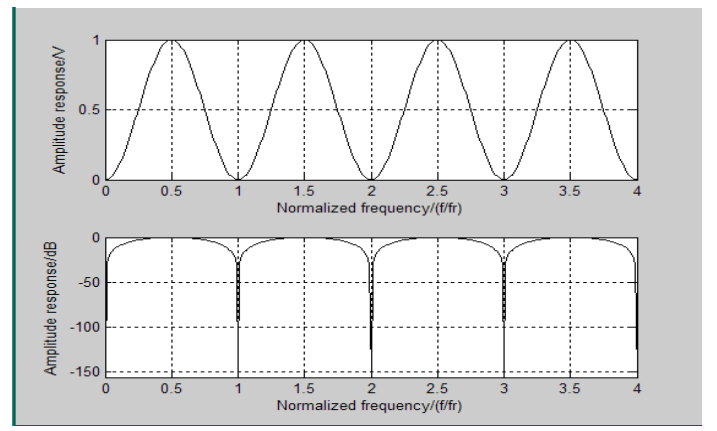

Figure 3. Normalized frequency response of single delay line cancellation

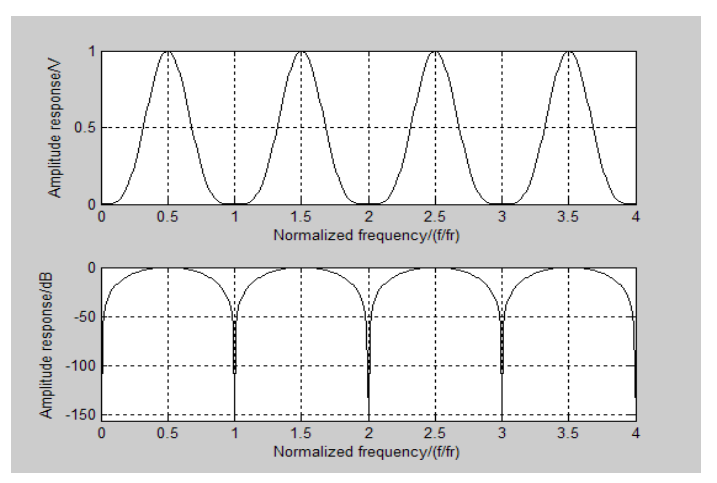

Figure 4. Normalized frequency response of double delay line cancellation

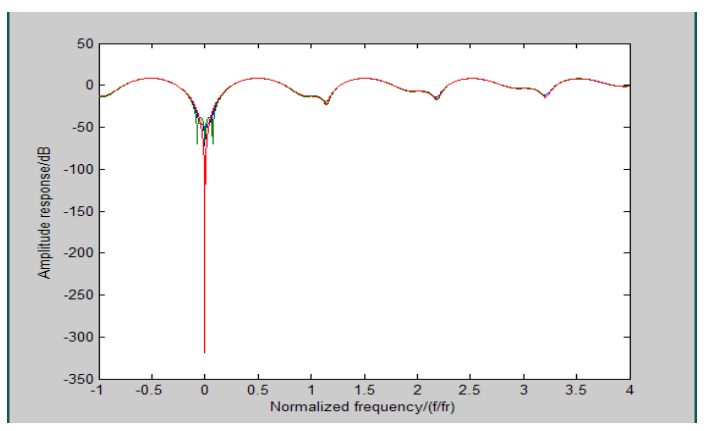

Figure 5. Single zero point FIR filter

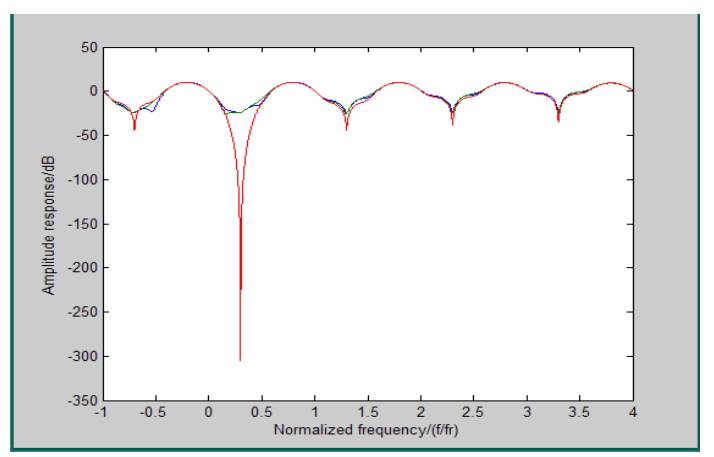

Figure 6. Multiple zero points FIR filter

\section{CONCLUSION}

From the above analysis, we can get that the delay line cancellation can only improve the zero Doppler frequency response, and to the reality of cloud clutter, the improvement is not ideal. Zero distribution method can be very good to make up for the shortage. And it enables the clutter to get maximum limit in the frequency points of the clutter 
and has the largest flat amplitude to other frequency ranges.

\section{REFERENCES}

[1] Huang Li, Research on echo generation of grand based radar and signal processing simulation. Cheng Du: University of electronic science and technology, 2006.

[2] Xing Mengdao et al, The foundation of radar signal processing. Beijing: Electronic industry press, 2012, 169171.
[3] Chen Boxiao et al, Analysis and design of modern radar systems. Xi'an: Xi'an electronic and science university press, 2012, 233-241.

[4] Long Fang et al, Research on MTI performance evaluation method of ground-based radar. Electronic countermeasures, 2002

[5] Zhang Juan, Simulation, evaluation and analysis of measured data of radar system. Xi'an: Xi'an electronic and science university press, 2006.

[6] Zhu Guofu et al, The Matlab simulation of radar system design. Beijing: Electronic industry press, 2009. 